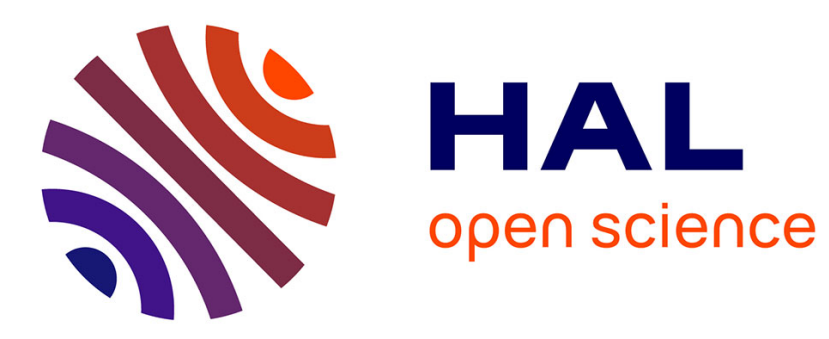

\title{
Les isolants du VIVITRON
}

J. Heugel, C. Brandt, M. Letournel, J.P. Resch, C. Roth, G. Sontag, H. Vogler, P. Wagner, A. Weber

\section{To cite this version:}

J. Heugel, C. Brandt, M. Letournel, J.P. Resch, C. Roth, et al.. Les isolants du VIVITRON. Accelerateurs Et Nouveaux Concepts D'Acceleration De Particules, 1987, Strasbourg, France. pp.1441-1443, 10.1051/rphysap:019880023090144100 . jpa-00245968

\section{HAL Id: jpa-00245968 https://hal.science/jpa-00245968}

Submitted on 1 Jan 1988

HAL is a multi-disciplinary open access archive for the deposit and dissemination of scientific research documents, whether they are published or not. The documents may come from teaching and research institutions in France or abroad, or from public or private research centers.
L'archive ouverte pluridisciplinaire HAL, est destinée au dépôt et à la diffusion de documents scientifiques de niveau recherche, publiés ou non, émanant des établissements d'enseignement et de recherche français ou étrangers, des laboratoires publics ou privés. 


\title{
Les isolants du VIVITRON
}

J. Heugel, C. Brandt, M. Letournel, J. P. Resch, C. Roth, G. Sontag, H. Vogler, P. Wagner et A. Weber

Centre de Recherches Nucléaires (IN2P3), 67037 Strasbourg Cedex, France

(Reçu le 15 octobre 1987, accepté le 17 mai 1988)

Résumé. - Les résultats de nombreux essais haute tension des différents isolants (composite verre-époxyde et plots) utilisés dans le VIVITRON sont reportés. Les tests de faisabilité d'une section accélératrice à l'échelle 1 sont décrits.

\begin{abstract}
The results of high voltage tests of the different types of insulators (composite material and post spacers) used in the VIVITRON are reported. A test generator representing one section of the new machine has been built and is described.
\end{abstract}

\section{Les isolants dans la technologie de construction.}

La structure isolante des accélérateurs électrostatiques est généralement réalisée avec des éléments subdivisés verre-métal. Rompant avec cette tradition, la construction du Vivitron fait appel à une technologie nouvelle utilisant deux types d'isolants.

Les isolants de structure matérialisant l'isolation longitudinale dans un champ électrique de $18 \mathrm{kV} / \mathrm{cm}$ sont constitués dans la construction traditionnelle par des poutres en verre-métal collées et assemblées par boulonnage. La structure nouvelle du Vivitron est proposée en matériau composite mat de verrerésine époxyde sous forme de deux poutres en treillis découpées dans des planches.

La construction traditionnelle est autosupportante et ne comporte pas d'éléments dans le champ électrique transversal (champ moyen de l'ordre de $130 \mathrm{kV} / \mathrm{cm})$. La nouvelle conception nécessite par contre un isolant support de structure. Cette isolation est matérialisée par des empilements d'isolateurs ou plots isolants en résine époxyde chargée de silice et d'alumine.

\section{Tests de faisabilité de la structure.}

\subsection{TEST DE DÉMONSTRATION.}

Conditions d'essai. — Ce test a permis de soumettre les composites proposés aux conditions réelles d'environnement d'une machine électrostatique. L'accélérateur Van de Graaff HVEC type CN 7 MV du CRN a été transformé pour tester en vraie grandeur une section Vivitron représentée par des barreaux isolants de 2,82 $\mathrm{m}$ de longueur. L'électrode terminale placée dans sa position d'origine est portée à l'aide de la courroie de Van de Graaff à une tension de 4,5 MV. Les isolants non subdivisés sont placés dans une zone où le champ est presque homogène (proche de $20 \mathrm{kV} / \mathrm{cm}$ ).

Résultats. - Le test diélectrique proprement dit de cet essai a permis de montrer qu'il était possible de maintenir une tension de 4,5 MV pendant un temps relativement long (quelques jours en continu, quelques semaines en discontinu) sur une grande longueur $(2,82 \mathrm{~m})$ de certains types de matériaux composites. Il a été possible de dépasser de $50 \%$ au moins cette valeur nominale correspondant à une section Vivitron, sans aucune perturbation ni dans le volume du matériau ni à la surface de ce dernier. Un essai comptabilisant 150 claquages au-dessus de $5 \mathrm{MV}$ a d'autre part clairement montré l'excellente tenue aux décharges du montage conçu pour laisser les isolants dans un environnement protégé.

\subsection{TEST DE CONFIRMATION.}

Conditions d'essai. - Un second test a été réalisé avec le prototype à l'échelle 1 d'une section accélératrice Vivitron. En effet, un montage intégrant deux planches en mat-époxyde comme éléments de structure, entourées de cinq électrodes coniques de colonne, a permis de remplacer la structure d'origine 
du générateur Van de Graaff CN 7 MV. L'électrode terminale d'origine a été modifiée pour harmoniser le champ électrique au niveau supérieur.

Résultats. - Sous une pression de 8 bars de SF6, ce montage a permis d'atteindre sans difficulté la tension nominale d'une section Vivitron (4,5 MV). Il a été possible d'y maintenir une tension supérieure à $5 \mathrm{MV}$ (avec un maximum de 5,6) pendant une semaine. Finalement, cet essai a permis de mettre définitivement au point plusieurs détails technologiques de montage et surtout de vérifier la validité des nouveaux concepts utilisés dans le projet Vivitron.

\section{Tests de mise au point des plots isolants.}

3.1 CARACTÉRISTIQUES DES PLOTS ISOLANTS. Les plots isolants proposés ont été développés par Cooke au High Voltage Research Laboratory du MIT, Cambridge, USA, dans le cadre des recherches concernant le transport de l'énergie à longue distance (câbles sous SF6).

Ces isolateurs de forme presque cylindrique, réalisés en résine époxyde chargée de silice et d'alumine, sont munis d'une électrode métallique (insert) à chaque extrémité. Ils supportent des tensions électriques relativement élevées. Dans l'application Vivitron, des plots isolants de $40 \mathrm{~cm}$ de long pour 4,5 MV sont placés entre deux électrodes dans un champ électrique divergent avec une contrainte assez élevée sur l'une des électrodes. La conception de ces plots permet d'avoir une distribution de champ plus homogène (Fig. 1).

Leur utilisation dans le Vivitron est le fruit d'une collaboration entre Cooke (MIT) et le CRN de Strasbourg. En effet, leur configuration définitive et

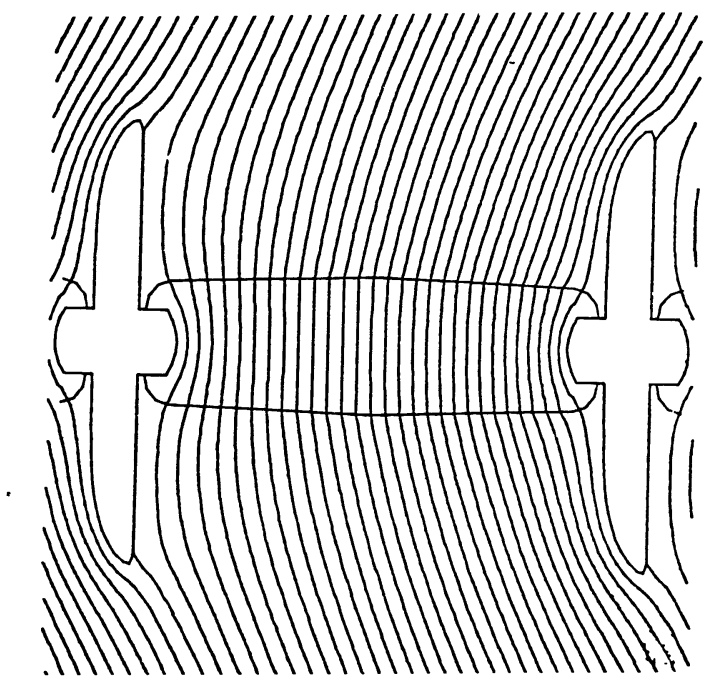

Fig. 1. - Tracé des équipotentielles autour d'un plot isolant monté entre deux électrodes discrètes.

[Equipotential lines around a post insulator mounted between to discrete electrodes.] leurs conditions d'utilisation ont été déterminées grâce à de nombreux essais effectués essentiellement avec le générateur Van de Graaff $\mathrm{CN} 7 \mathrm{MV}$.

3.2 ESSAIS DE TENUE À LA HAUTE TENSION ET AUX CLAQUAGES. - Un programme d'essais H.T. dans des conditions de champ électrique assez sévères par rapport au projet a été entrepris en utilisant les accélérateurs $\mathrm{CN}$ et $\mathrm{MP}$ du CRN ainsi que la machine pilote du laboratoire de Daresbury, Warrington, GB. Plusieurs dizaines de plots ont ainsi été testées en fonction de différents paramètres, permettant de cumuler plus de 1000 heures-plot sous haute tension particulièrement au niveau 4,5 MV, mais également à $5 \mathrm{MV}$ et même jusqu'à $6 \mathrm{MV}$. Durant ces essais environ $30 \%$ des plots ont subi des dommages, la moitié de ceux-ci restant inutilisables.

De nombreux enseignements ont été tirés de ces tests :

- la tenue en tension est indépendante du type d'insert ;

- la tenue en tension n'est pas altérée par la contrainte mécanique que l'isolateur doit supporter ;

- la détection des plots défectueux (défauts de fabrication) se fait à une tension inférieure à 4,5 MV;

- le bon comportement H.T. des plots nécessite l'élimination de l'humidité introduite dans l'isolant par la mise à l'air ;

- la pression de fonctionnement optimale est de 8 bars de SF6.

Finalement le comportement global permet de conclure qu'un plot de $40 \mathrm{~cm}$ de long tient facilement 4,5 MV avec cependant quelques restrictions. En effet les claquages risquent de marquer la surface de l'isolant (dégradation de la résine par carbonisation). Il apparaît qu'un nombre réduit de claquages n'affecte pas la tenue en tension. Par conséquent il est important de placer les plots dans un environnement les protégeant des décharges (Fig. 2).

3.3 ESSAIS DE LONGUE DURÉE. ESSAIS DE RÉCEPTION. - Plusieurs types d'essais H.T., toujours avec le générateur Van de Graaff CN 7 MV du CRN, ont permis de poursuivre l'étude du comportement des plots Vivitron. La disposition et la forme des électrodes H.T. ont été choisies pour permettre de tester plusieurs plots en même temps et pour approcher au plus près les conditions de champ électrique Vivitron. Plus particulièrement, un essai de longue durée (39 jours) a consisté à maintenir 3 plots pendant 663 heures sans interruption sous haute tension, c'est-à-dire :

$$
\begin{gathered}
30 \% \text { du temps entre } 4,5 \text { et } 5 \mathrm{MV} \\
17 \% \text { du temps entre } 5 \text { et } 5,3 \mathrm{MV} \\
6 \% \text { du temps entre } 5,3 \text { et } 5,6 \mathrm{MV} \text {, }
\end{gathered}
$$

le reste du temps étant attribué au fonctionnement 


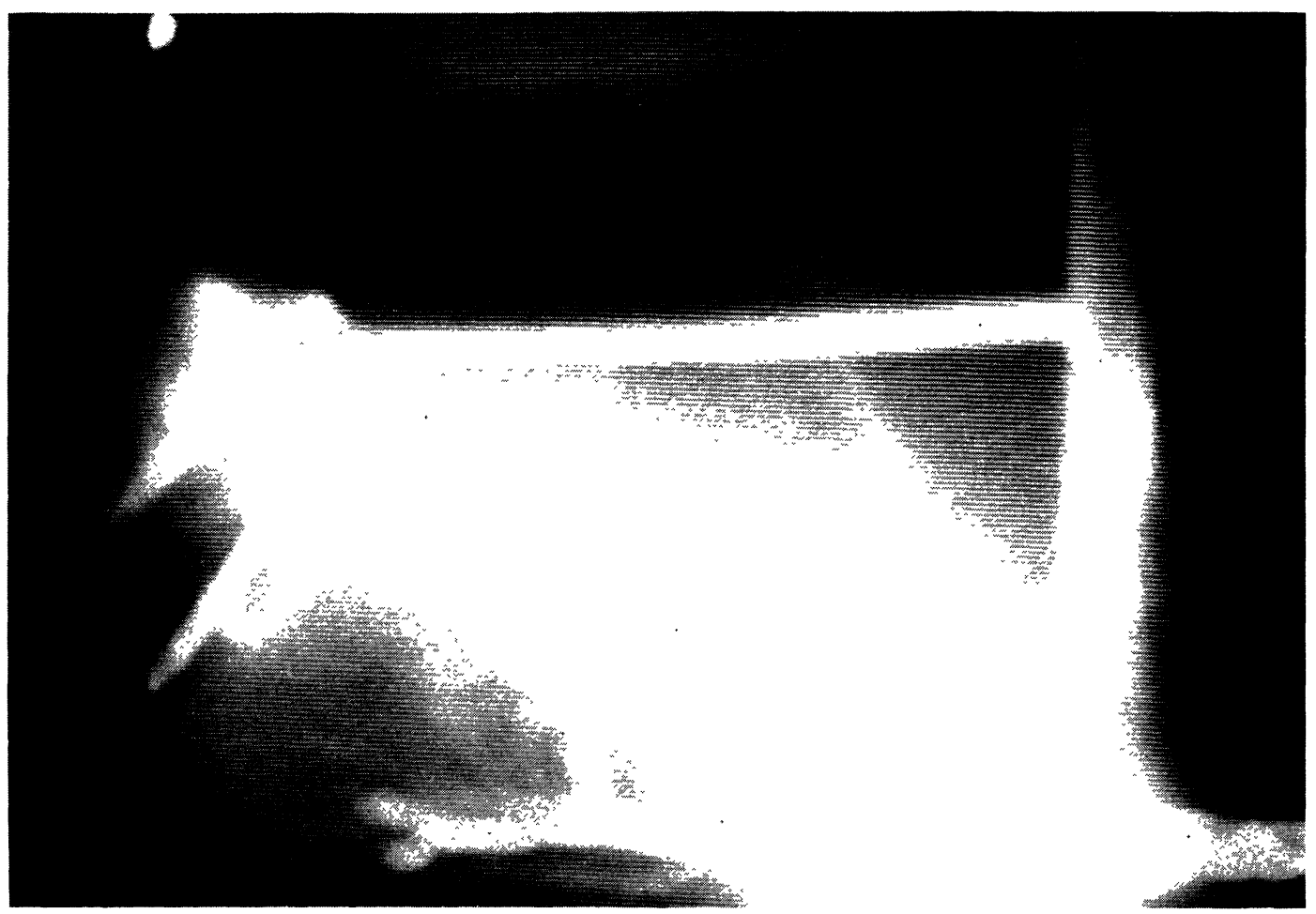

Fig. 2. - Claquage à 5,65 MV autour d'un plot de $40 \mathrm{~cm}$ monté entre l'électrode H.T. du générateur CN $7 \mathrm{MV}$ (côté droit) et une contre-électrode (côté gauche). L'observation est faite avec une caméra de télévision.

[Spark at a voltage of $5.65 \mathrm{MV}$ near a post of $40 \mathrm{~cm}$ length and installed between two electrodes.]

de nuit à un niveau dépassant 1,5 MV. Au total une trentaine de claquages isolés ont été comptabilisés. Ils n'ont eu aucune conséquence sur l'état des plots ou leur tenue à la haute tension.

Il faut encore signaler qu'un certain nombre d'essais ont montré que la restauration par sablage à l'alumine était parfaitement valable pour des plots marqués en surface par des décharges.
Les essais de plots Vivitron se poursuivent encore. En effet les plots livrés pour monter le Vivitron, subissent tous un test de réception à $10 \%$ au-dessus de la tension nominale. D'autre part, on ne peut manquer de mentionner les essais faits avec des isolateurs de fabrication française, très prometteurs, et qui devraient remplacer dans un avenir proche les plots américains en cas de besoin. 